International Journal of Pure and Applied Mathematics

Volume 88 No. 3 2013, 351-362

ISSN: 1311-8080 (printed version); ISSN: 1314-3395 (on-line version)

url: http://www.ijpam.eu

doi: http://dx.doi.org/10.12732/ijpam.v88i3.3

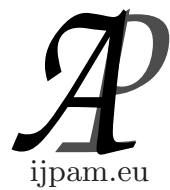

\title{
SOME PROPERTIES OF $N(k)$-QUASI EINSTEIN MANIFOLDS
}

\author{
Jaeman Kim \\ Department of Mathematics Education \\ Kangwon National University \\ Chunchon, 200-701, KOREA
}

\begin{abstract}
In this paper, an example of an $N(k)$-quasi Einstein manifold with closed associated 1-form is given. Also we show that if a quasi Einstein manifold $\left(M_{1}^{n_{1}}, g_{1}\right)$ and an Einstein manifold $\left(M_{2}^{n_{2}}, g_{2}\right)$ satisfy a certain condition, then the Riemannian product manifold $\left(M^{n}, g\right)=\left(M_{1}^{n_{1}} \times M_{2}^{n_{2}}, g_{1}+g_{2}\right)$ is a quasi Einstein manifold. In particular, in $N(k)$-quasi Einstein case, we show that there exists a quasi Einstein product manifold $\left(M^{n}, g\right)=\left(M_{1}^{n_{1}} \times M_{2}^{n_{2}}, g_{1}+\right.$ $\left.g_{2}\right)$ but not an $N(k)$-quasi Einstein manifold, which consists of an $N(k)$-quasi Einstein manifold $\left(M_{1}^{n_{1}}, g_{1}\right)$ and an Einstein manifold $\left(M_{2}^{n_{2}}, g_{2}\right)$ satisfying the certain condition. Finally we study an $N(k)$-quasi Einstein manifold satisfying the condition $R(U, X) \cdot G=0$.
\end{abstract}

AMS Subject Classification: 53A30, 53A40, 53B20

Key Words: quasi Einstein manifold, associated 1-form, $N(k)$-quasi Einstein manifold, Riemannian product manifolds, quasi Einstein product manifold, Gcurvature tensor, Killing vector field, Ricci-semisymmetric manifold

\section{Introduction}

A non-flat Riemannian manifold $\left(M^{n}, g\right)$ of dimension $n \geq 3$ is said to be a quasi Einstein manifold if the Ricci tensor $r$ of $\left(M^{n}, g\right)$ is not identically zero

Received: May 16, 2013

(c) 2013 Academic Publications, Ltd. url: www.acadpubl.eu 
and satisfies the condition

$$
r(X, Y)=a g(X, Y)+b u(X) u(Y)
$$

for some smooth functions $a$ and $b \neq 0$, where $u$ is a non-zero 1-form such that

$$
g(X, U)=u(X), g(U, U)=u(U)=1
$$

for the associated unit vector field $U$. The 1 -form $u$ is called the associated 1 -form and the unit vector field $U$ is called the generator of the quasi Einstein manifold $\left(M^{n}, g\right)$. The notion of quasi Einstein manifolds arose during the study of exact solutions of the Einstein field equations as well as during considerations of quasi umbilical hypersurfaces. Investigations in [2] and others have revealed that a conformally flat quasi Einstein manifold has the geometric structure of quasi constant curvature. Also it has been found that a manifold of quasi constant constant is a natural subclass of quasi Einstein manifold [4].

Let $R$ denote the Riemannian curvature tensor of $\left(M^{n}, g\right)$. The $k$-nullity distribution $N(k)$ of $\left(M^{n}, g\right)$ is defined for all $X, Y \in T_{p} M^{n}$ by

$$
N(k): p \mapsto N_{p}(k)=\left\{Z \in T_{p} M^{n}: R(X, Y) Z=k(g(Y, Z) X-g(X, Z) Y)\right\},
$$

where $k$ is a smooth function. If the generator $U$ of a quasi Einstein manifold $\left(M^{n}, g\right)$ belongs to the $k$-nullity distribution $N(k)$, then the quasi Einstein manifold $\left(M^{n}, g\right)$ is called as an $N(k)$-quasi Einstein manifold. In [12], it was shown that a conformally flat quasi Einstein manifold is an $N(k)$-quasi Einstein manifold and in particular, a quasi Einstein manifold of dimension 3 is an $N(k)$ quasi Einstein manifold.

In [5], [6] and [7], Pokhariyal and Mishra introduced a type of curvaturelike tensor called $G$-curvature tensor and studied its relativistic significance. According to them, a $G$-curvature tensor $G$ on a Riemannian manifold $\left(M^{n}, g\right)$ is defined by

$$
\begin{gathered}
G(X, Y, Z, V)=R(X, Y, Z, V)-\frac{1}{2(n-1)}(g(X, Z) r(Y, V)-g(X, V) r(Y, Z) \\
-g(Y, Z) r(X, V)+g(Y, V) r(X, Z)) .
\end{gathered}
$$

In this connection, they introduced a $W^{*}$-curvature tensor defined on the line of Weyl projective curvature tensor. The $G$-curvature tensor $G$ has been defined by breaking $W^{*}$ into skew-symmetric parts [7].

In this paper, we give an example of an $N(k)$-quasi Einstein manifold with closed associated 1-form. In case of Riemannian product manifolds, we show 
that if a quasi Einstein manifold $\left(M_{1}^{n_{1}}, g_{1}\right)$ and an Einstein manifold $\left(M_{2}^{n_{2}}, g_{2}\right)$ satisfy the condition $n_{2} a_{1}=s_{2}$, then the Riemannian product manifold $\left(M^{n}, g\right)$ of $\left(M_{1}^{n_{1}}, g_{1}\right)$ and $\left(M_{2}^{n_{2}}, g_{2}\right)$ is a quasi Einstein manifold. Therefore we can raise a natural question whether or not the Riemannian product manifold $\left(M^{n}, g\right)=$ $\left(M_{1}^{n_{1}} \times M_{2}^{n_{2}}, g_{1}+g_{2}\right)$ is an $N(k)$-quasi Einstein manifold if the quasi Einstein manifold $\left(M_{1}^{n_{1}}, g_{1}\right)$ from the above mentioned statement is in fact an $N(k)$-quasi Einstein manifold. This paper gives a negative answer of this question. Finally an $N(k)$-quasi Einstein manifold satisfying the condition $R(U, X) \cdot G=0$ has been studied.

\section{Preliminaries}

Let $\left(M^{n}, g\right)$ be a Riemannian manifold. The Riemannian curvature tensor $R$, Ricci tensor $r$ and scalar curvature $s$ are defined by

$$
R(X, Y) Z=\nabla_{X} \nabla_{Y} Z-\nabla_{Y} \nabla_{X} Z-\nabla_{[X, Y]} Z
$$

And

$$
\begin{gathered}
R(X, Y, Z, W)=g(R(X, Y) Z, W) . \\
r(Y, Z)=\sum_{i=1, \ldots, n} R\left(e_{i}, Y, Z, e_{i}\right), \\
s=\sum_{i=1, \ldots, n} r\left(e_{i}, e_{i}\right),
\end{gathered}
$$

where $\left\{e_{i}\right\}_{i=1, . ., n}$ is an orthonormal frame. The Riemannian manifold $\left(M^{n}, g\right)$ is called an Einstein manifold if the Ricci tensor $r$ is proportional to the metric tensor $g$, i.e., $r=\frac{s}{n} g$. The Riemannian curvature tensor $R$ has the following well known $S O(n)$-decomposition [1];

$$
R=\frac{s}{2 n(n-1)} g \bullet g+\frac{1}{n-2} r_{o} \bullet g+W,
$$

where $r_{o}$ is the traceless Ricci tensor and $W$ is the Weyl curvature tensor. Here the symbol $\bullet$ is the Nomizu-Kulkarni product of symmetric (0,2)-tensors generating a curvature type tensor:

$$
\begin{aligned}
h \bullet k(X, Y, Z, V)=h(X, Z) k(Y, V)+ & h(Y, V) k(X, Z) \\
& -h(X, V) k(Y, Z)-h(Y, Z) k(X, V) .
\end{aligned}
$$


Note that $r_{o}=0$ if and only if $\left(M^{n}, g\right)$ is Einstein, and $W=0$ if and only if $\left(M^{n}, g\right)$ is conformally flat. The Weyl curvature tensor depends only on the conformal class of $\left(M^{n}, g\right)$. Moreover, it satisfies the curvature symmetries and so we can treat it as a conformal curvature tensor. Note that the Weyl curvature tensor is traceless.

For a $(0, k)$-tensor field $A$ on $M^{n}$, we define a $(0, k+2)$-tensor field $R \cdot A$ (see [10] and [11]) by

$$
\begin{aligned}
& (R \cdot A)\left(X_{1}, \ldots, X_{k} ; X, Y\right)=(R(X, Y) \cdot A)\left(X_{1}, \ldots, X_{k}\right) \\
& \quad=-A\left(R(X, Y) X_{1}, X_{2}, \ldots, X_{k}\right)-\ldots-A\left(X_{1}, \ldots, X_{k-1}, R(X, Y) X_{k}\right) .
\end{aligned}
$$

If a Riemannian manifold $\left(M^{n}, g\right)$ satisfies the condition $R \cdot R=0$ (resp. $\left.R \cdot r=0\right)$, then $\left(M^{n}, g\right)$ is said to be semisymmetry (resp. Ricci-semisymmetry) (see [10] and [11]). It is well known that the class of semisymmetric manifolds includes the set of locally symmetric manifolds $(\nabla R=0)$ as a proper subset and that the class of Ricci -semisymmetric manifolds includes the set of Ricci-symmetric manifolds $(\nabla r=0)$ as a proper subset (see [10] and [11]).

In a quasi Einstein manifold $\left(M^{n}, g\right)$ if the generator $U$ belongs to some $k$-nullity distribution $N(k)$, then we get

$$
R(X, Y) U=k(u(Y) X-u(X) Y),
$$

which is equivalent to

$$
R(X, U) Y=k(u(Y) X-g(X, Y) U)=-R(U, X) Y .
$$

Moreover in [8] and [12], for an $N(k)$-quasi Einstein manifold $\left(M^{n}, g\right)$

$$
k=\frac{a+b}{n-1} .
$$

\section{Product Manifolds and $N(k)$-Quasi Einstein Manifolds}

In this section, we investigate some relations between product manifolds and $N(k)$-quasi Einstein manifolds. First of all, we give an example of an $N(k)$-quasi Einstein manifold with closed associated 1-form.

Example 3.1. Let $\left(R^{n}, g\right)(n \geq 3)$ be a Riemannian manifold endowed with the metric $g$ given by

$$
g=e^{2 x_{1}}\left(d x_{1}^{2}+d x_{2}^{2}+\cdots+d x_{n}^{2}\right) .
$$


It is easy to see that the Riemannian manifold $\left(M^{n}, g\right)$ is conformally flat. From (2.5), it follows that on $\left(R^{n}, g\right)$

$$
r_{11}=2-n
$$

and

$$
r_{i j}=0
$$

otherwise, where $r_{i j}=r\left(\frac{\partial}{\partial x_{i}}, \frac{\partial}{\partial x_{j}}\right)$.

Therefore we get

$$
r=0 g+(2-n) e^{-2 x_{1}} u \otimes u,
$$

where $u=e^{x_{1}} d x_{1}$. And hence $\left(R^{n}, g\right)$ is a quasi Einstein manifold with $a=0$ and $b=(2-n) e^{-2 x_{1}} \neq 0$. In fact, $\left(R^{n}, g\right)$ is an $N(k)$-quasi Einstein manifold because $\left(R^{n}, g\right)$ is conformally flat. Furthermore, it is easy to see that

$$
d u=0 .
$$

Summing up the above arguments, we obtain the following: $R^{n}$ allows a Riemannian metric $g$ such that $\left(R^{n}, g\right)$ is an $N(k)$-quasi Einstein manifold whose associated 1-form $u$ is closed.

Concerning Riemannian product manifolds, we have

Theorem 3.2. Let $\left(M_{1}^{n_{1}}, g_{1}\right)$ and $\left(M_{2}^{n_{2}}, g_{2}\right)$ be a quasi Einstein manifold and an Einstein manifold, respectively. Then the Riemannian product manifold $\left(M^{n}, g\right)=\left(M_{1}^{n_{1}} \times M_{2}^{n_{2}}, g_{1}+g_{2}\right)$ is a quasi Einstein manifold if the following condition

$$
n_{2} a_{1}=s_{2}
$$

is satisfied. (Here $a_{1}$ is the associated scalar of a quasi Einstein manifold $\left(M_{1}^{n_{1}}, g_{1}\right)$ and $s_{2}$ is the scalar curvature of an Einstein manifold $\left.\left(M_{2}^{n_{2}}, g_{2}\right)\right)$

Proof. Since $\left(M_{1}^{n_{1}}, g_{1}\right)$ and $\left(M_{2}^{n_{2}}, g_{2}\right)$ are a quasi Einstein manifold and an Einstein manifold, respectively, we get

$$
r_{1}=a_{1} g_{1}+b_{1} u \otimes u
$$

and

$$
r_{2}=\frac{s_{2}}{n_{2}} g_{2} .
$$

By virtue of the condition $n_{2} a_{1}=s_{2}$, the Riemannian product manifold $\left(M^{n}, g\right)$ $=\left(M_{1}^{n_{1}} \times M_{2}^{n_{2}}, g_{1}+g_{2}\right)$ has its Ricci tensor $r$ as

$$
r=r_{1}+r_{2}=a_{1}\left(g_{1}+g_{2}\right)+b_{1} u \otimes u
$$




$$
=a_{1} g+b_{1} u \otimes u,
$$

which implies that $\left(M^{n}, g\right)$ is a quasi Einstein manifold. This completes the proof of theorem 3.1 .

In case of $N(k)$-quasi Einstein manifolds, we are able to show that the $N(k)$-quasi Einstein version of Theorem 3.1 does not hold true:

Example 3.3. Let $\left(R^{3}, g_{1}\right)$ be a conformally flat manifold endowed with the metric $g_{1}$ given by

$$
g_{1}=e^{2 x_{1}}\left(d x_{1}^{2}+d x_{2}^{2}+d x_{3}^{2}\right) .
$$

From the previous example, it follows that $\left(R^{3}, g_{1}\right)$ is an $N(k)$-quasi Einstein manifold. More precisely, its Ricci tensor $r_{1}$ satisfies the relation

$$
r_{1}=0 g_{1}+\left(-e^{-2 x_{1}}\right) u \otimes u,
$$

where $a_{1}=0, b_{1}=-e^{-2 x_{1}} \neq 0$ and $u=e^{x_{1}} d x_{1}$. On the other hand, let $\left(R^{n-3}, g_{2}\right)$ be a standard flat manifold with the metric $g_{2}$ given by

$$
g_{2}=d x_{4}^{2}+d x_{5}^{2}+\cdots+d x_{n}^{2} .
$$

It is obvious that its Ricci tensor $r_{2}$ satisfies the relation

$$
r_{2}=0 g_{2},
$$

which implies that $\left(R^{n-3}, g_{2}\right)$ is an Einstein manifold with $s_{2}=0$. Therefore the Riemannian product manifold $\left(R^{n}, g\right)=\left(R^{3} \times R^{n-3}, g_{1}+g_{2}\right)$ is a quasi Einstein manifold since the condition (3.12) is satisfied. Note that the generator of the quasi Einstein product manifold $\left(R^{n}, g\right)=\left(R^{3} \times R^{n-3}, g_{1}+g_{2}\right)$ is given by

$$
U=e^{-x_{1}} \frac{\partial}{\partial x_{1}} .
$$

However the quasi Einstein product manifold $\left(R^{n}, g\right)=\left(R^{3} \times R^{n-3}, g_{1}+g_{2}\right)$ is not an $N(k)$-quasi Einstein manifold. In fact, if we assume that the Riemannian product manifold $\left(R^{n}, g\right)=\left(R^{3} \times R^{n-3}, g_{1}+g_{2}\right)$ is an $N(k)$-quasi Einstein manifold, then taking account of $(2.4),(2.9)$ and (2.11), we have

$$
\begin{aligned}
& R\left(\frac{\partial}{\partial x_{1}}, \frac{\partial}{\partial x_{4}}, \frac{\partial}{\partial x_{1}}, \frac{\partial}{\partial x_{4}}\right) \\
& \quad=\frac{-e^{-2 x_{1}}}{n-1}\left[g\left(\frac{\partial}{\partial x_{1}}, \frac{\partial}{\partial x_{4}}\right) g\left(\frac{\partial}{\partial x_{1}}, \frac{\partial}{\partial x_{4}}\right)-g\left(\frac{\partial}{\partial x_{1}}, \frac{\partial}{\partial x_{1}}\right) g\left(\frac{\partial}{\partial x_{4}}, \frac{\partial}{\partial x_{4}}\right)\right]
\end{aligned}
$$




$$
=\frac{1}{n-1}
$$

On the other hand, from the definition of Riemannian product manifold, it follows that

$$
\begin{aligned}
R\left(\frac{\partial}{\partial x_{1}}, \frac{\partial}{\partial x_{4}}, \frac{\partial}{\partial x_{1}}, \frac{\partial}{\partial x_{4}}\right) & \\
& =R_{1}\left(\frac{\partial}{\partial x_{1}}, \frac{\partial}{\partial x_{4}}, \frac{\partial}{\partial x_{1}}, \frac{\partial}{\partial x_{4}}\right)+R_{2}\left(\frac{\partial}{\partial x_{1}}, \frac{\partial}{\partial x_{4}}, \frac{\partial}{\partial x_{1}}, \frac{\partial}{\partial x_{4}}\right)=0
\end{aligned}
$$

where $R_{1}$ and $R_{2}$ are the Riemannian curvature tensors of $\left(R^{3}, g_{1}\right)$ and $\left(R^{n-3}, g_{2}\right)$, respectively. This is a contradiction. Summing up the above arguments, we obtain the following: There exists a quasi Einstein product manifold $\left(R^{n}, g\right)=$ $\left(R^{3} \times R^{n-3}, g_{1}+g_{2}\right)$ but not an $N(k)$-quasi Einstein manifold, which consists of an $N(k)$-quasi Einstein manifold $\left(R^{3}, g_{1}\right)$ and an Einstein manifold $\left(R^{n-3}, g_{2}\right)$ satisfying the condition (3.12).

\section{4. $N(k)$-Quasi Einstein Manifold Satisfying $R(U, X) \cdot G=0$}

In this section, we consider an $N(k)$-quasi Einstein manifold satisfying the condition $R(U, X) \cdot G=0$. First of all, we have

Lemma 4.1. In an $N(k)$-quasi Einstein manifold $\left(M^{n}, g\right)$, the $G$-curvature tensor satisfies the relation

$$
G(X, Y, Z, U)=\frac{4 a+3 b}{2(n-1)}(u(X) g(Y, Z)-u(Y) g(X, Z))
$$

for all vector fields $X, Y, Z$ on $M^{n}$.

Proof. Taking account of (1.1),(1.2),(2.9) and (2.11), we have

$$
\begin{gathered}
G(X, Y, Z, U)=R(X, Y, Z, U)-\frac{1}{2(n-1)}[g(X, Z) r(Y, U)- \\
-g(X, U) r(Y, Z)-g(Y, Z) r(X, U)+g(Y, U) r(X, Z)] \\
=\frac{a+b}{n-1}[u(X) g(Y, Z)-u(Y) g(X, Z)]-\frac{1}{2(n-1)}[g(X, Z)(a g(Y, U)+b u(Y) u(U)) \\
-g(X, U)(a g(Y, Z)+b u(Y) u(Z))-g(Y, Z)(a g(X, U)+b u(X) u(U))+ \\
+g(Y, U)(a g(X, Z)+b u(X) u(Z))]
\end{gathered}
$$




$$
=\frac{4 a+3 b}{2(n-1)}(u(X) g(Y, Z)-u(Y) g(X, Z)) .
$$

This completes the proof of Lemma 4.1.

Now we give the main results of this section.

Theorem 4.2. Let $\left(M^{n}, g\right)$ be an $N(k)$-quasi Einstein manifold. If $\left(M^{n}, g\right)$ satisfies the condition $R(U, X) \cdot G=0$, then $a+b=0$.

Proof. Assume that $\left(M^{n}, g\right)$ is an $N(k)$-quasi Einstein manifold and satisfies the condition $R(U, X) \cdot G=0$. Then from (2.8) we can write

$$
\begin{aligned}
0=G(R(U, X) Y, Z, V, W) & +G(Y, R(U, X) Z, V, W)+G(Y, Z, R(U, X) V, W)+ \\
& +G(Y, Z, V, R(U, X) W)
\end{aligned}
$$

From (2.10) and (4.14), it follows that

$$
\begin{aligned}
0 & =\frac{a+b}{n-1}[G(g(X, Y) U-g(U, Y) X, Z, V, W)+G(Y, g(X, Z) U-g(U, Z) X, V, W)+ \\
& +G(Y, Z, g(X, V) U-g(U, V) X, W)+G(Y, Z, V, g(X, W) U-g(U, W) X)] .
\end{aligned}
$$

From the above identity, it follows that

$$
\begin{gathered}
0=\frac{a+b}{n-1}[g(X, Y) G(U, Z, V, W)-g(U, Y) G(X, Z, V, W)+ \\
+g(X, Z) G(Y, U, V, W)-g(U, Z) G(Y, X, V, W)+g(X, V) G(Y, Z, U, W)- \\
-g(U, V) G(Y, Z, X, W)+g(X, W) G(Y, Z, V, U)-g(U, W) G(Y, Z, V, X)] .
\end{gathered}
$$

Substituting $W=U$ into the above identity, we get either

$$
a+b=0
$$

or

$$
\begin{gathered}
0=g(X, Y) G(U, Z, V, U)-g(U, Y) G(X, Z, V, U)+ \\
+g(X, Z) G(Y, U, V, U)-g(U, Z) G(Y, X, V, U)+g(X, V) G(Y, Z, U, U)- \\
-g(U, V) G(Y, Z, X, U)+g(X, U) G(Y, Z, V, U)-g(U, U) G(Y, Z, V, X) .
\end{gathered}
$$

Now assume that $a+b \neq 0$. Taking account of (4.13) and (4.16), we have

$$
G(Y, Z, V, X)=\frac{4 a+3 b}{2(n-1)}(g(X, Y) g(Z, V)-g(X, Z) g(Y, V)) .
$$


By virtue of (1.2), we have

$$
\begin{aligned}
R(Y, Z, V, X)= & \frac{4 a+3 b}{2(n-1)}(g(X, Y) g(Z, V)-g(X, Z) g(Y, V)) \\
& +\frac{1}{2(n-1)}(g(Y, V) r(Z, X) \\
& -g(Y, X) r(Z, V)-g(Z, V) r(Y, X)+g(Z, X) r(Y, V))
\end{aligned}
$$

Contracting (4.17) over $X$ and $Y$, we get

$$
r(Z, V)=\frac{(4 a+3 b)(n-1)-s}{3 n-4} g(Z, V),
$$

which contradicts our assumption that $\left(M^{n}, g\right)$ is an $N(k)$-quasi Einstein manifold. Hence we can conclude that $\left(M^{n}, g\right)$ satisfies the condition $a+b=0$. This completes the proof of Theorem 4.2.

As a consequence we obtain

Corollary 4.3. Let $\left(M^{n}, g\right)$ be an $N(k)$-quasi Einstein manifold. If $\left(M^{n}, g\right)$ satisfies the condition $R(U, X) \cdot G=0$, then $R(X, Y, Z, U)=0$.

Proof. By virtue of Theorem 4.2 we have

$$
a+b=0,
$$

which implies from (2.4),(2.9) and (2.11) that

$$
R(X, Y, Z, U)=0 .
$$

This completes the proof.

Let $C$ denote the symmetric endomorphism of $T_{p} M^{n}$ corresponding to the Ricci tensor $r$, that is, $g(C X, Y)=r(X, Y)$, where $X, Y \in T_{p} M^{n}$. The transformations $R(X, Y)$ and $C$ are called the curvature transformation and the Ricci transformation, respectively. It is known that in a quasi Einstein manifold the curvature and the Ricci transformations commute if and only if the relation $R(X, Y, Z, U)=0$ holds [3]. According to this fact we get the following:

Corollary 4.4. Let $\left(M^{n}, g\right)$ be an $N(k)$-quasi Einstein manifold. If $\left(M^{n}, g\right)$ satisfies the condition $R(U, X) \cdot G=0$, then the curvature and the Ricci transformations commute. 
In the compact case, if we assume that the generator $U$ is a Killing vector field, then we are able to show that $U$ ia a parallel vector field. More precisely we have

Theorem 4.5. Let $\left(M^{n}, g\right)$ be a compact $N(k)$-quasi Einstein manifold with Killing vector field $U$. If $\left(M^{n}, g\right)$ satisfies the condition $R(U, X) \cdot G=0$, then $U$ is a parallel vector field.

Proof. It is known that for a vector field $X$ on a compact Riemannian manifold $M^{n}$ the following relation holds [9]:

$$
\int_{M^{n}}\left(r(X, X)-|\nabla X|^{2}-(\operatorname{div} X)^{2}\right) d M \leq 0 .
$$

and equality holds if and only if $X$ is a Killing vector field [9]. Moreover if $X$ is a Killing vector field then $\operatorname{div} X=0$. Therefore the above inequality takes the following form:

$$
\int_{M^{n}}\left(r(X, X)-|\nabla X|^{2}\right) d M=0 .
$$

In case of a Killing vector field $U$, the above identity yields by virtue of $(2.4),(2.5),(2.9)$ and $(2.11)$ that

$$
\int_{M^{n}}\left((a+b)-|\nabla U|^{2}\right) d M=0 .
$$

From Theorem 4.2 and the last identity, it follows that

$$
\nabla U=0 .
$$

This completes the proof of Theorem 4.5.

According to Theorem 4.5 we obtain the following:

Corollary 4.6. Let $\left(M^{n}, g\right)$ be a compact $N(k)$-quasi Einstein manifold with Killing vector field $U$. If $\left(M^{n}, g\right)$ satisfies the condition $R(U, X) \cdot G=0$, then $\left(M^{n}, g\right)$ is a product manifold.

Proof. Since the generator $U$ is a Killing vector field we have

$$
g\left(\nabla_{X} U, Y\right)+g\left(\nabla_{Y} U, X\right)
$$

for every $X, Y \in T_{p} M^{n}$. Now let $U^{\perp}$ denote the $(n-1)$-dimensional distribution in $\left(M^{n}, g\right)$ orthogonal to $U$ and if $X$ and $Y$ belong to $U^{\perp}$ then

$$
g(X, U)=g(Y, U)=0 .
$$


From $\left(\nabla_{X} g\right)(Y, U)=0$ and Theorem 4.5, it follows that

$$
0=g\left(\nabla_{X} Y, U\right)+g\left(Y, \nabla_{X} U\right)=g\left(\nabla_{X} Y, U\right) .
$$

Similarly we have

$$
g\left(\nabla_{Y} X, U\right)=0
$$

Therefore since the Riemannian connection $\nabla$ is of vanishing torsion, that is,

$$
[X, Y]=\nabla_{X} Y-\nabla_{Y} X
$$

we obtain that

$$
g([X, Y], U)=g\left(\nabla_{X} Y-\nabla_{Y} X, U\right)=0,
$$

which means $[X, Y] \in U^{\perp}$ for every $X, Y \in U^{\perp}$. From the Frobenius Theorem, it follows that $U^{\perp}$ is integrable. Hence $\left(M^{n}, g\right)$ is a product manifold. This completes the proof.

Due to Theorem 4.5 we also have

Corollary 4.7. Let $\left(M^{n}, g\right)$ be a compact $N(k)$-quasi Einstein manifold with Killing vector field $U$. If $\left(M^{n}, g\right)$ satisfies the condition $R(U, X) \cdot G=0$, then $\left(M^{n}, g\right)$ is a Ricci-semisymmetric manifold.

Proof. In virtue of $a+b=0$, we have

$$
r=a g+b u \otimes u=a(g-u \otimes u) .
$$

From (4.18), $\nabla U=0$ and $a=-b \neq 0$, it follows that

$$
\nabla r=d a(g-u \otimes u)=\frac{d a}{a} r=(d \log a) r .
$$

Taking account of (4.19), we have

$$
\left(\nabla_{X} \nabla_{Y} r\right)(V, W)=(X(d \log a)(Y)+(d \log a)(X)(d \log a)(Y)) r(V, W),
$$

which yields from (2.3)

$$
(R(X, Y) \cdot r)(V, W)=2 d(d \log a)(X, Y) r(V, W)=0 .
$$

Hence we conclude that $\left(M^{n}, g\right)$ is a Ricci-semisymmetric manifold. This completes the proof. 


\section{Acknowledgments}

This study is supported by Kangwon National University.

\section{References}

[1] A.L. Besse, Einstein Manifolds, Springer, Berlin (1987).

[2] M.C. Chaki, M.L. Ghosh, On quasi Einstein manifolds, Ind. Jour. Math. 42 (2000), 211-220.

[3] M.C. Chaki, R.K. Maity, On quasi Einstein manifold, Publ. Math. Debrecen, 57 (2000), 297-306.

[4] U.C. De, G.C. Ghosh, On quasi Einstein manifolds, Period. Math. Hungar., 48 (2004), 223-231, doi: 10.1023/B:MAHU.0000038977.94711.ab.

[5] G.P. Pokhariyal, R.S. Mishra, Curvature tensors and their relativistic significance, Yokohama Math. J., 18 (1970), 105-108.

[6] G.P. Pokhariyal, R.S. Mishra, Curvature tensors and their relativistic significance II, Yokohama Math. J., 19, No. 2 (1971), 97-103.

[7] G.P. Pokhariyal, Curvature tensors and their relativistic significance III, Yokohama Math. J., 21 (1973), 115-119.

[8] C. Ozgur, $N(k)$-quasi Einstein manifolds satisfying certain conditions, Chaos, Solitons and Fractals, 38 (2008), 1373-1377.

[9] A.A. Shaikh, On pseudo quasi Einstein manifolds, Period. Math. Hungar., 59 (2009), 119-146, doi: 10.1007/s10998-009-0119-6.

[10] Z.I. Szabo, Structure theorems on Riemannian spaces satisfying $R(X, Y)$. $R=0$ I, the local version, J. Diff. Geometry, 17 (1982), 531-582.

[11] Z.I. Szabo, Structure theorems on Riemannian spaces satisfying $R(X, Y)$. $R=0$ II, Global versions, Geom. Dedicata, 19 (1985), 65-108.

[12] M.M. Tripathi, J.S. Kim, On $N(k)$-quasi Einstein manifolds, Commun. Korean Math. Soc., 22 (2007), 411-417, doi: 10.4134/CKM.2007.22.3.411. 